\title{
Relationship between outpatient visit frequency and hypertension control: a 9-year occupational cohort study
}

\author{
Azusa Shima ${ }^{1,2}$, Yukako Tatsumi ${ }^{1,3}$, Tatsuro Ishizaki ${ }^{4}$, Kayo Godai ${ }^{1}$, Yuichiro Kawatsu ${ }^{2,5}$, Tomonori Okamura ${ }^{6}$, \\ Tomofumi Nishikawa ${ }^{7}$, Akiko Morimoto ${ }^{1}$, Ayumi Morino ${ }^{1}$ and Naomi Miyamatsu ${ }^{1}$ \\ The purpose of this study was to investigate the relationship between the frequency of outpatient visits and hypertension \\ control as determined from health insurance records. This 9-year cohort study in Japan was based on 518 participants with \\ hypertension who underwent health checkups in 2004. Participants were aged 35-56 years and none had a history of \\ cardiovascular or cerebrovascular disease. All were covered by the same employee health insurer. Mean annual outpatient visit \\ days at a hospital/clinic during the 9-year period were classified within four quartiles (Q1, Q2, Q3, Q4). Uncontrolled \\ hypertension was defined as a systolic blood pressure (BP) $\geqslant 140 \mathrm{~mm} \mathrm{Hg}$ and a diastolic BP $\geqslant 90 \mathrm{~mm} \mathrm{Hg}$. Logistic regression \\ analysis was used to estimate the multivariable-adjusted odds ratios (ORs) and $95 \%$ confidence intervals (Cls) for the prevalence \\ of uncontrolled hypertension in groups Q1, Q2 and Q3 vs. Q4. The median (25th-75th percentile) annual outpatient visit days \\ was 9.4 (4.0-15.5). Uncontrolled hypertension was observed in $62.4 \%$ of the participants in 2013. The multivariable-adjusted \\ ORs and 95\% Cls for uncontrolled hypertension in Q1, Q2 and Q3 vs. Q4 were 4.03 (2.28-7.12), $1.67(0.99-2.81)$ and \\ $1.44(0.86-2.41)$, respectively. Uncontrolled hypertension increased significantly as the number of outpatient visits decreased \\ $(P$ for trend $<0.001)$. This tendency was maintained when participants taking antihypertensive agents at baseline were \\ excluded. Our study showed an inverse relationship between outpatient visit frequency and uncontrolled hypertension. \\ Hypertension Research (2016) 39, 376-381; doi:10.1038/hr.2015.157; published online 14 January 2016
}

Keywords: health checkup; health insurance; hypertension control; outpatient visit frequency

\section{INTRODUCTION}

Hypertension is a major risk factor for cardiovascular disease. Many studies have shown that the risk of cardiovascular morbidity and mortality increases with hypertension, irrespective of age, ${ }^{1,2}$ and that life expectancy decreases with increased blood pressure (BP) levels. ${ }^{3}$ Most clinical trials have clearly shown that a reduction in BP decreases the incidence of stroke and heart disease ${ }^{4-6}$ and that the benefits could be explained by changes in BP alone. ${ }^{6}$ However, in many parts of the world, including Japan, hypertension is not adequately controlled. ${ }^{7-11}$ In addition to the adverse effects on the physical health of patients, several Japanese epidemiological studies suggested that uncontrolled hypertension led to an increase in medical expenditures. ${ }^{12-15}$

In Japan, employees are required by the Industrial Safety and Health Act to undergo annual health checkups that include BP measurements. ${ }^{16,17}$ Thus hypertension is often identified during these examinations. However, Tanaka et al. ${ }^{18}$ showed that the proportion of Japanese workers in their 40 s who had hypertension for which they were taking antihypertensive agents was only $27.9 \%$ in males and $32.3 \%$ in females.

In the medical setting, a positive relationship between outpatient visit frequency and hypertension control is expected, as patients who frequently visit outpatient clinics might be more health-conscious and the frequent patient-physician contact would favor timely initiation of treatment. Japanese guidelines recommend antihypertensive drug therapy for patients whose BP cannot be reduced to $140 / 90 \mathrm{~mm} \mathrm{Hg}$ within 3 months through lifestyle modifications alone. ${ }^{19}$ Physicians can then adjust the medications of patients who frequently return to the clinic, resulting in better BP control. Indeed, several studies have reported an association between the frequency of outpatients' visits, whether to a hospital or a clinic, and hypertension control among patients already started on antihypertension therapy in Europe and the United States. ${ }^{20-23}$ However, to our knowledge, no prospective studies have investigated the relationship between outpatient visit frequency and hypertension control among Japanese people. In Japan, under the

${ }^{1}$ Department of Clinical Nursing, Shiga University of Medical Science, Shiga, Japan; ${ }^{2}$ Occupational Health Care Office, Heiwado Co., Ltd., Shiga, Japan; ${ }^{3}$ Department of Preventive Medicine and Epidemiology, National Cerebral and Cardiovascular Center, Osaka, Japan; ${ }^{4}$ Human Care Research Team, Tokyo Metropolitan Institute of Gerontology, Tokyo, Japan; ${ }^{5}$ Department of Health Policy and Management, Institute of Industrial Ecological Sciences, University of Occupational and Environmental Health, Kitakyushu, Japan; ${ }_{6}^{6}$ Preventive Medicine and Public Health, Keio University, Tokyo, Japan and ${ }^{7}$ Department of Health and Nutrition, Kyoto Koka Women's University, Kyoto, Japan Correspondence: A Shima, Department of Clinical Nursing, Shiga University of Medical Science, Seta Tsukinowa-cho, Otsu, Shiga 520-2192, Japan.

E-mail: shiazusa@belle.shiga-med.ac.jp

Received 9 July 2015; revised 28 November 2015; accepted 30 November 2015; published online 14 January 2016 
universal health insurance system, the fees for medical services and drugs are set by the government. ${ }^{24,25} \mathrm{We}$ were therefore able to assess the relationship without taking into account different insurers and medical institutions. In the present study, we investigated the association between outpatient visit frequency over 9 years and BP control among Japanese retail employees with hypertension who were insured by an Employees' Health Insurance Society.

\section{METHODS}

\section{Japanese health insurance system}

In Japan, under the universal coverage principal of 1961, all residents are required to enroll in a health insurance system. Residents aged $<75$ years are covered by Employees' Health Insurance $(58 \%)$ or by the National Health Insurance (31\%), according to their occupation. Residents aged $>75$ years are covered by the Advanced Elderly Medical Service (coverage rate $11 \%$ ). Employees' Health Insurance is for salaried workers and their dependent family members. Beneficiaries may visit any hospital or clinic granted approval. Thus, in Japan, there is a universal health insurance coverage and access based on free choice. Fees for medical services at all approved hospitals and clinics are strictly controlled by the National Government and are paid on a fee-for-service basis. When a beneficiary uses a medical service, he or she pays $30 \%$ of the cost, whereas the insurance organization pays $70 \%$, with no taxes imposed. Information on the number of outpatient visits, medical fees and the medical services are required to be recorded monthly in an insurance claim history file. ${ }^{24,25}$ Data on the number of outpatient visit days evaluated in the present study were extracted from insurance claim data.

\section{Study setting}

The study population consisted of full-time and part-time employees aged $20-65$ years who worked $\geqslant 6 \mathrm{~h}$ a day at a retail company with locations across nine prefectures (Hyogo, Osaka, Kyoto, Shiga, Fukui, Ishikawa, Toyama, Gifu and Aichi) in Japan. Most of the participants underwent annual health checkups at their workplaces. These were performed using standardized methods in accordance with the Industrial Safety and Health Act. ${ }^{16}$ Health checkup items do not differ between full-time and part-time employees. Employees with health problems detected during the health checkup are encouraged to visit a clinic or hospital.

All study participants were covered by the same Employees' Health Insurance Society, which is independent of the retail company where they were employed. Annual health checkup data and health insurance claim data are recorded by the insurer. By the end of March 2014, about 15000 employees were registered in its database, which is annually updated. For this study, anonymized data were provided to Shiga University of Medical Science. Participants were informed about the opportunity to opt-out of this study on the intranet page of the Employees' Health Insurance Society. The present study was conducted in accordance with the Ethics Committee of Shiga University of Medical Science (24-18).

\section{Participants}

The enrolled subjects were 2977 participants aged 35-56 years who underwent a health checkup in 2004 (baseline health checkup) and continued their work until 2013 (9 years). Individuals aged $\geqslant 57$ years were excluded as they would have reached the retirement age of 65 years before the end of the observation period in 2013. In 2004, 554 participants were identified as having hypertension, as defined by the following criteria: (1) systolic blood pressure (SBP) $\geqslant 140 \mathrm{~mm} \mathrm{Hg}$, (2) diastolic blood pressure (DBP) $\geqslant 90 \mathrm{~mm} \mathrm{Hg}$, and (3) the use of antihypertensive agents, according to the guidelines of the Japanese Society of Hypertension. ${ }^{19}$ Participants were excluded if they had a history of cardiovascular or cerebrovascular disease $(n=12)$, missing data $(n=14)$ or did not undergo a health checkup in $2013(n=10)$. Thus the final study population consisted of 518 participants with hypertension.

\section{Health checkups}

Baseline checkups were carried out from January to March 2004. Health checkups included anthropometry measurements, BP measurements, blood tests, a urine dipstick test, electrocardiogram, chest X-ray and self-administered questionnaires on health-related habits and medical histories. BP was measured with participants in the sitting position and was recorded by trained nurses using electronic sphygmomanometers. BP was measured a second time if the participant's SBP was $\geqslant 140 \mathrm{~mm} \mathrm{Hg}$ or DBP was $\geqslant 90 \mathrm{~mm} \mathrm{Hg}$. The mean value was then used in the analyses. Blood tests included determination of serum levels of total cholesterol and glucose. Hyperglycemia was defined as a plasma glucose level $\geqslant 140 \mathrm{mg} \mathrm{dl}^{-1}$ or treatment for diabetes (using antidiabetic agents or insulin) and hypercholesterolemia as a total cholesterol level $\geqslant 220 \mathrm{mg} \mathrm{dl}^{-1}$ or the use of cholesterol-lowering agents. Prior to the blood tests, $80 \%$ of participants had not fasted. Height and weight were measured with the participant clothed. Body mass index was calculated as weight in kilograms divided by the square of the height in meters. Data collected in a self-administered questionnaire included medical history, smoking habit (non-smoker, quit smoking and current smoker) and drinking habit (non-drinker, occasional drinker and daily drinker).

\section{Outpatient visit frequency}

The number of outpatient visit days of each participant from January 2004 to December 2012 was extracted from health insurance claim data. Data for inpatient and dental care were not included. The mean number of annual outpatient visit days was calculated as the total outpatient visit days from 2004 to 2012 divided by 9 years.

\section{Definition of outcome and follow-up}

Participants were followed until 2013. Annual health checkups were performed every January to March using the same procedure. The presence of uncontrolled hypertension was determined at the participant's health checkup in 2013. Uncontrolled hypertension was defined as $\mathrm{SBP} \geqslant 140 \mathrm{~mm} \mathrm{Hg}$ or $\mathrm{DBP} \geqslant 90 \mathrm{~mm} \mathrm{Hg}$ regardless of the use of antihypertensive agents. ${ }^{19}$

\section{Statistical analysis}

To analyze outpatient visit frequency as a categorical variable, four quartiles representing mean annual outpatient visit days were established. To compare the baseline characteristics of participants assigned to the quartiles, analysis of covariance was used for continuous variables and $\chi^{2}$ test for dichotomous and categorical variables. The proportions of participants who, in 2013, were taking antihypertensive agents, cholesterol-lowering agents or insulin or antihyperglycemic agents for the treatment of diabetes were calculated using the information provided in the questionnaires.

Logistic regression analysis was used to estimate the multivariable-adjusted odds ratios (ORs) and 95\% confidence intervals (CIs) for the prevalence of uncontrolled hypertension in groups Q1, Q2 and Q3 compared with group Q4 (most frequent outpatient visits). Model 1 was adjusted for sex and age, model 2 for sex, age, body mass index, DBP, smoking status and drinking status and model 3 for the variables in model 2 plus the presence or absence of hypercholesterolemia, hyperglycemia and urinary protein. The DBP was adjusted to account for the different values among the quartiles.

A further analysis was conducted after excluding participants who were taking antihypertensive agents at baseline, because their outpatient visit frequency might have been influenced by the dosing period of the antihypertensive agents. In addition, we compared the effect of the outpatient visit frequency of the former (2004-2008) and latter (2009-2012) halves of the observational period on the prevalence of uncontrolled hypertension in 2013.

SPSS 20.0 for Windows (SPSS Japan, Tokyo, Japan) was used for the statistical analyses. All $P$-values were two-tailed. $P<0.05$ was considered to indicate statistical significance.

\section{RESULTS}

The mean age (s.d.) of the participants was 47.5 (5.1) years, and $64.7 \%$ were female. The median (25th-75th percentile) number of annual outpatient visit days was 9.4 (range: $4.0-15.5$ ). The proportion of participants taking antihypertensive agents was $27.2 \%$ at baseline and $66.4 \%$ in 2013. 
Table 1 Outpatient visit days per year and characteristics of participants at baseline among quartiles

\begin{tabular}{|c|c|c|c|c|c|}
\hline & Q1 (0.0-4.0) & Q2 (4.1-9.3) & Q3 (9.4-15.4) & Q4 (15.6-156.3) & P-value \\
\hline No. of participants & 133 & 125 & 131 & 129 & \\
\hline Men, $n(\%)^{\mathrm{a}}$ & $62(46.7)$ & $47(37.6)$ & $36(27.5)$ & $38(29.5)$ & $<0.001$ \\
\hline Age (years) & $46.5(45.7-47.3)$ & $46.7(45.9-47.5)$ & $48.2(47.4-49.0)$ & $48.8(48.0-49.6)$ & $<0.001$ \\
\hline Outpatient visit days, median (25-75th percentiles) & $1.9(1.1-3.2)$ & $7.1(5.5-8.2)$ & $12.0(10.6-13.9)$ & $20.7(18.1-25.7)$ & \\
\hline Body mass index $\left(\mathrm{kg} \mathrm{m}^{-2}\right)^{\mathrm{c}}$ & $23.9(23.2-24.6)$ & $24.6(23.9-25.3)$ & $25.4(24.7-26.1)$ & $24.7(24.1-25.4)$ & 0.03 \\
\hline \multicolumn{6}{|l|}{ Blood pressure $(m m ~ H g)^{c}$} \\
\hline Systolic & $146.8(144.2-149.4)$ & $147.7(145-150.3)$ & $150.6(148.0-153.2)$ & $147.2(144.5-149.8)$ & 0.16 \\
\hline Diastolic & $90.0(88.3-91.7)$ & $90.6(88.8-92.4)$ & $93.6(91.9-95.3)$ & $89.6(87.9-91.4)$ & 0.01 \\
\hline Antihypertensive medication, $n(\%)^{a}$ & $2(1.6)$ & $15(12.0)$ & $57(43.6)$ & $67(52.0)$ & $<0.001$ \\
\hline Blood glucose $\left(\mathrm{mg} \mathrm{dl}^{-1}\right)^{\mathrm{c}}$ & $105.2(99.2-111.3)$ & $102.1(95.9-108.3)$ & $113.3(107.2-119.3)$ & $109.7(103.6-115.8)$ & 0.06 \\
\hline High blood glucose, $n(\%)^{a, d}$ & $9(6.8)$ & $5(4.0)$ & $16(12.3)$ & $19(14.7)$ & 0.01 \\
\hline Diabetes treatment, $n(\%)^{a, e}$ & $1(0.8)$ & $0(0.0)$ & $6(4.6)$ & $7(5.4)$ & 0.01 \\
\hline Total cholesterol $\left(\mathrm{mg} \mathrm{dl}^{-1}\right)^{\mathrm{c}}$ & $211.9(206.1-217.7)$ & $213.4(207.5-219.3)$ & $214.2(208.4-220)$ & $214(208.2-219.9)$ & 0.95 \\
\hline Hypercholesterolemia, $n(\%)^{a, f}$ & $57(42.9)$ & $53(42.4)$ & $60(45.9)$ & $58(45.0)$ & 0.94 \\
\hline Hypercholesterolemia medication, $n(\%)^{\mathrm{a}}$ & $0(0.0)$ & $1(0.8)$ & $3(2.3)$ & $7(5.4)$ & 0.01 \\
\hline Uric protein, $n(\%)^{\mathrm{a}}$ & $7(5.3)$ & $8(6.4)$ & $5(3.9)$ & $9(7.0)$ & 0.70 \\
\hline \multicolumn{6}{|l|}{ Smoking habit, $n(\%)^{\mathrm{a}}$} \\
\hline Non-smoker & $74(55.7)$ & $70(56.0)$ & $79(60.4)$ & 87 (67.5) & 0.15 \\
\hline Quit smoking & $9(6.8)$ & $14(11.2)$ & $11(8.4)$ & $14(10.9)$ & \\
\hline Current smoker & $50(37.6)$ & $41(32.8)$ & $41(31.3)$ & $28(21.8)$ & \\
\hline \multicolumn{6}{|l|}{ Drinking habit, $n(\%)^{\mathrm{a}}$} \\
\hline Non-drinker & $49(36.9)$ & $59(47.2)$ & $57(43.6)$ & $46(35.7)$ & 0.28 \\
\hline Occasional drinker & $43(32.4)$ & $33(26.4)$ & $42(32.1)$ & $51(39.6)$ & \\
\hline Daily drinker & $41(30.9)$ & $33(26.4)$ & $32(24.5)$ & $32(24.9)$ & \\
\hline
\end{tabular}

Age is indicated as mean with $95 \%$ confidence interval adjusted for sex by analysis of covariance.

${ }^{a}$ Categorical values are indicated as number (percentage) analyzed by chi-square test.

bOutpatient visit days was the mean number of outpatient visit days per year calculated as total outpatient visit days from 2004 to 2012 divided by 9 .

${ }^{c}$ Mean with $95 \%$ confidence interval, sex and age adjusted by analysis of covariance.

dDefined as a plasma glucose level $\geqslant 140 \mathrm{mg} \mathrm{dl}^{-1}$ or use of antihyperglycemic agents.

eDiabetes treatment, including antihyperglycemic agents or insulin.

fDefined as total cholesterol level $\geqslant 220 \mathrm{mg} \mathrm{dl}^{-1}$ or use of cholesterol-lowering agents.

Table 1 shows the characteristics of the study participants according to the quartiles of number of outpatient visit days per year. The outpatient visit days of group Q4 were widely distributed, with 25th and 75th percentiles of 18.1 and 25.7 days, respectively. Participants with a high frequency of outpatient visits were significantly more likely to be female and older. Mean DBP but not mean SBP levels were significantly different between quartiles. The proportion of participants taking antihypertensive agents increased significantly from Q1 to Q4. Mean blood glucose levels were higher in Q3 and Q4 than in Q1 and Q2, whereas there were no differences in mean total cholesterol levels, the prevalence of hypercholesterolemia, smoking status and drinking status.

Data on the use of medication for hypertension and other cardiovascular risks in 2013 are shown in Table 2. The proportion of study participants taking antihypertensive agents increased in all quartiles, although the percentage was $>85 \%$ in both Q3 and Q4 but only $22.6 \%$ in Q1. The proportion of participants taking cholesterollowering agents and receiving treatment for diabetes increased with increasing outpatient visit days among all participants, including those who were not taking antihypertensive agents at the 2004 baseline.

Uncontrolled hypertension was observed in 323 (62.4\%) participants in 2013. Table 3 shows the prevalence of uncontrolled hypertension and the multivariable-adjusted ORs and 95\% CIs for uncontrolled hypertension among quartiles. The prevalence of uncontrolled hypertension was 79.0\% in Q1, 60.8\% in Q2, 59.6\% in Q3 and $49.7 \%$ in Q4. The multivariable-adjusted ORs and 95\% CIs of uncontrolled hypertension in Q1, Q2 and Q3 compared with Q4 were 4.03 (2.28-7.12), $1.67(0.99-2.81)$ and $1.44(0.86-2.41)$, respectively. The prevalence of uncontrolled hypertension increased significantly with decreasing outpatient visit frequency $(P$ for trend $<0.001)$. The difference between the quartiles was less marked when we used SBP as a covariate instead of DBP.

In a sensitivity analysis, the same analysis was conducted except that the 141 participants taking antihypertensive agents at baseline were excluded. This allowed assessment of the effect of outpatient visits on the initiation of hypertension treatment. The multivariable-adjusted ORs and 95\% CIs after the exclusion of these participants was 4.76 (2.39-9.52) in Q1, $1.79(0.92-3.47)$ in Q2 and $1.87(0.91-3.85)$ in Q3 $(P<0.001$ for trend $)$.

Both in the first 5 years (2004-2008) and last 4 years (2009-2012) of the study, the prevalence of uncontrolled hypertension significantly increased with decreasing outpatient visit frequency $(P<0.001$ for trend). However, the multivariable-adjusted OR and 95\% CI of Q1 were larger in the last 4 years than in the first 5 years (Table 4 ). One hundred and thirty participants were in the highest quartile (Q4) during the first 5 years (2004-2008). Of these, 38, 8 and 1 patient came under Q3, Q2 and Q1, respectively, in the last 4 years (2009-2012). 
Table 2 Proportion of participants taking medication for hypertension or other cardiovascular risk factors in 2013

\begin{tabular}{|c|c|c|c|c|}
\hline Outpatient visit frequency & $\mathrm{n}$ & Antihypertensive medication (\%) & Cholesterol-lowering medication (\%) & Diabetes treatment $(\%)^{n}$ \\
\hline \multicolumn{5}{|l|}{ All participants } \\
\hline Q1 (0.0-4.0) & 133 & $30(22.6)$ & $9(6.8)$ & $3(2.3)$ \\
\hline Q3 (9.4-15.4) & 131 & $115(87.8)$ & $41(31.3)$ & $13(9.9)$ \\
\hline Q4 (15.6-156.3) & 129 & $111(86.0)$ & $49(38.0)$ & $18(14.0)$ \\
\hline$P$-value ${ }^{\mathrm{b}}$ & & $<0.001$ & $<0.001$ & 0.001 \\
\hline Q1 (0.0-4.0) & 131 & $29(22.1)$ & $8(6.1)$ & $3(2.3)$ \\
\hline Q2 (4.1-9.3) & 110 & $74(67.3)$ & $18(16.4)$ & $9(8.2)$ \\
\hline Q3 (9.4-15.4) & 74 & $61(82.4)$ & $26(35.1)$ & $10(13.5)$ \\
\hline Q4 (15.6-156.3) & 62 & $47(75.8)$ & $19(30.6)$ & $9(14.5)$ \\
\hline$P$-value ${ }^{b}$ & & $<0.001$ & $<0.001$ & 0.001 \\
\hline
\end{tabular}

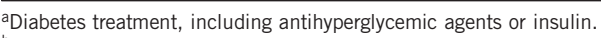

${ }^{b}$ Chi-square test.

Table 3 Multivariable-adjusted ORs and 95\% Cls for uncontrolled hypertension among quartiles after 9 years

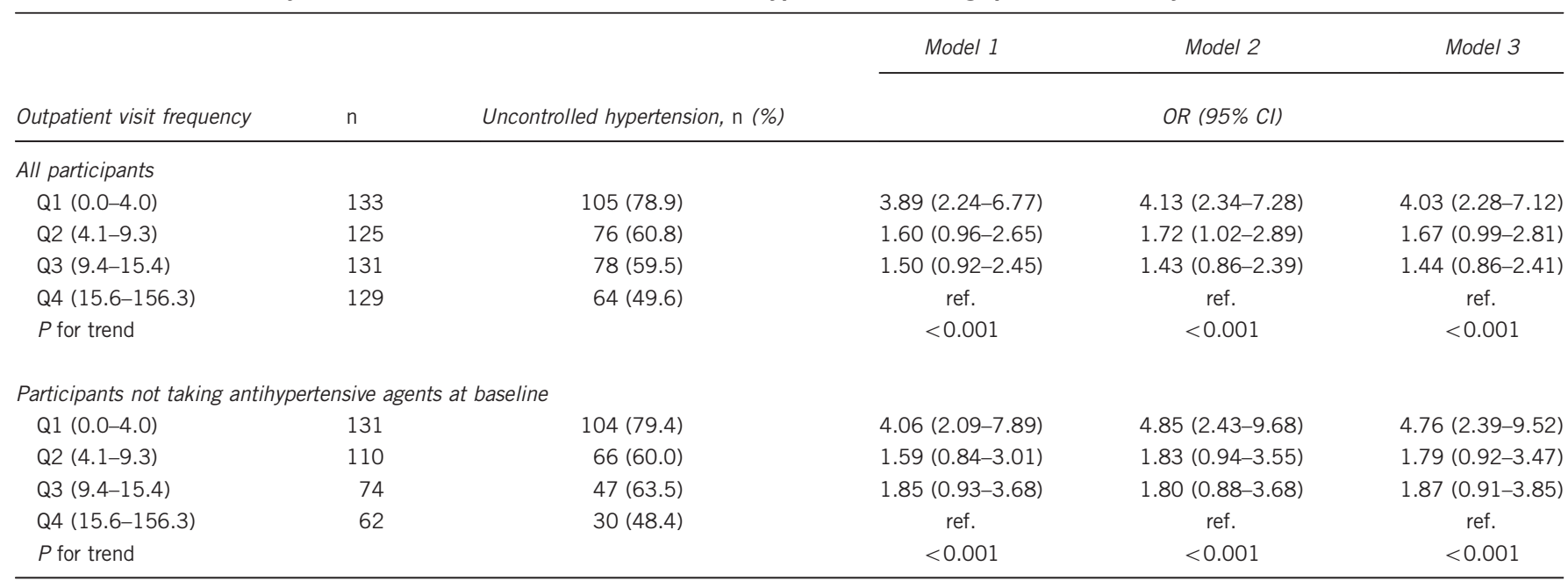

Abbreviations: $\mathrm{Cl}$, confidence interval; $\mathrm{OR}$, odds ratio.

Uncontrolled hypertension was defined as SBP $\geqslant 140 \mathrm{~mm} \mathrm{Hg}$ or DBP $\geqslant 90 \mathrm{~mm} \mathrm{Hg}$

Logistic regression analysis was used to estimate the adjusted OR and $95 \% \mathrm{Cls}$.

Model 1: Adjusted for sex and age.

Model 2: Adjusted items in Model 1 plus diastolic blood pressure, body mass index, smoking habit and drinking habit.

Model 3: Adjusted all items in Model 2 plus hypercholesterolemia, hyperglycemia and urinary protein.

Finally, the outpatient frequency during the first 5 years (2004-2008) was also associated with the prevalence of uncontrolled hypertension in 2009 (data not shown).

\section{DISCUSSION}

In this 9-year cohort study, we investigated the relationship between outpatient visit frequency, as determined from health insurance claim data, and consequent hypertension control among participants with hypertension. Antihypertensive agents were taken by $66.4 \%$ of the participants and the prevalence of uncontrolled hypertension at 9 years was $62.4 \%$. The multivariable-adjusted OR for uncontrolled hypertension was approximately four times higher in group with few outpatient visits (Q1) than in the group with highest number of visits (Q4, once or twice a month). This tendency did not change when participants with baseline use of antihypertensive agents was excluded.

The association between the frequency of clinic visits and hypertension control has been the subject of several studies, which have yielded conflicting results. In their study of 113 patients visiting clinics in the
US state of Oklahoma, Parchman et al. ${ }^{20}$ did not find an association between visit frequency and hypertension control, whereas in an observational study involving 429 patients at two urban family practice centers in the United States, a shorter return visit interval was associated with better changes in BP ((final BP-initial BP)/initial BP). ${ }^{21}$ Recently, a retrospective cohort study of 5042 hypertensive patients with diabetes mellitus attending clinics affiliated with two academic medical centers in Massachusetts demonstrated an association between shorter encounter intervals, a faster decrease in BP and earlier BP normalization..$^{22}$ In the same retrospective study, in a cohort of 26496 patients with diabetes mellitus, consultation with a primarycare provider every 2 weeks was associated with the fastest achievement of hemoglobin $\mathrm{A}_{10}, \mathrm{BP}$ and low-density lipoprotein cholesterol targets. ${ }^{23}$ The results of the present study are consistent with those findings. The distinctive aspect of our study was that it used health insurance claims data to classify outpatient visit frequency. Thus participants were not limited to particular hospitals or clinics and included those who had not been taking antihypertensive agents at baseline. 
Table 4 Multivariable-adjusted ORs and 95\% Cls for uncontrolled hypertension in 2013 among quartiles in the first 5 years and last 4 years

\begin{tabular}{|c|c|c|c|c|c|c|}
\hline \multirow[b]{2}{*}{ Outpatient visit frequency } & \multirow[b]{2}{*}{$\mathrm{n}$} & \multirow{2}{*}{$\begin{array}{l}\text { Median of outpatient } \\
\text { visit days per year }\end{array}$} & \multirow{2}{*}{$\begin{array}{l}\text { Uncontrolled hypertension, } \\
\qquad \mathrm{n}(\%)\end{array}$} & Model 1 & Model 2 & Model 3 \\
\hline & & & & \multicolumn{3}{|c|}{ OR $(95 \% \mathrm{Cl})$} \\
\hline \multicolumn{7}{|c|}{ In the first 5 years (2004-2008) } \\
\hline Q1 (0.0-2.6) & 132 & $1.2(0.4-2.0)$ & $94(71.2)$ & $2.20(1.31-3.71)$ & $2.24(1.31-3.81)$ & $2.17(1.27-3.71)$ \\
\hline Q2 (2.8-8.0) & 127 & $5.0(3.6-6.2)$ & $87(68.5)$ & $1.93(1.15-3.24)$ & $2.08(1.22-3.53)$ & $2.04(1.20-3.47)$ \\
\hline Q3 (8.2-14.8) & 129 & $11.0(9.4-13.1)$ & $73(56.6)$ & $1.15(0.71-1.89)$ & $1.10(0.66-1.82)$ & $1.08(0.64-1.80)$ \\
\hline$P$ for trend & & & & 0.001 & $<0.001$ & $<0.001$ \\
\hline \multicolumn{7}{|c|}{ In the last 4 years (2009-2012) } \\
\hline Q1 $(0.0-4.8)$ & 128 & $2.0(0.8-3.0)$ & $105(82.0)$ & $4.33(2.42-7.73)$ & $4.73(2.60-8.58)$ & $4.60(2.53-8.37)$ \\
\hline Q2 (5.0-10.8) & 129 & $8.0(6.3-9.8)$ & $72(55.8)$ & $1.19(0.72-1.95)$ & $1.21(0.72-2.01)$ & $1.16(0.69-1.95)$ \\
\hline Q3 (11.0-16.5) & 130 & $13.4(12.3-14.8)$ & $78(60.0)$ & $1.39(0.85-2.27)$ & $1.39(0.84-2.32)$ & $1.39(0.83-2.32)$ \\
\hline
\end{tabular}

Abbreviations: $\mathrm{Cl}$, confidence interval; OR, odds ratio.

Uncontrolled hypertension was defined as SBP $\geqslant 140 \mathrm{~mm} \mathrm{Hg}$ or DBP $\geqslant 90 \mathrm{~mm} \mathrm{Hg}$ at 2013.

Logistic regression analysis was used to estimate the adjusted OR and $95 \% \mathrm{Cls}$.

Model 1: Adjusted for sex and age.

Model 2: Adjusted items in Model 1 plus diastolic blood pressure, body mass index, smoking habit and drinking habit.

Model 3: Adjusted all items in Model 2 plus hypercholesterolemia, hyperglycemia and urinary protein.

The positive association between outpatient visit frequency and hypertension control determined in this study can be explained as follows. The group with frequent visits had a higher proportion of participants who were taking antihypertensive agents both at baseline and at 9 years. In Japan, patients on antihypertensive agents are usually required to visit an outpatient clinic within 3 months. Participants in Q2-Q4 visited outpatient clinics more than once every 3 months and would have been more likely to receive effective treatment and appropriate changes in their medication. By contrast, in Q1, with fewer outpatient visits, the prevalence of uncontrolled hypertension tended to be higher.

The association of outpatient frequency with uncontrolled hypertension did not change when participants already taking antihypertensive agents at baseline were excluded, which suggests that for patients frequently visiting outpatient clinics, physicians can prescribe medications at the appropriate time and adjust them as needed to achieve better BP control. The proportion of participants receiving treatment for hyperlipidemia and hyperglycemia also increased with increasing outpatient visits. Japanese clinical guidelines for the treatment of hypertension, dyslipidemia and diabetes require physicians to comprehensively control possible cardiovascular risk factors. ${ }^{19,26,27}$ Therefore, frequent outpatient visits may lead to better control of hypertension, even if the main purpose of the outpatient visit was for other chronic diseases that may affect or be affected by hypertension.

The frequency of outpatient visits may also have been associated with non-pharmacological treatment or with a patient's desire for treatment, given that the proportions of participants on antihypertensive medication did not differ between Q3 and Q4. Participants who frequently visited outpatient clinics may be more health-conscious and likely to follow the recommendations of their physicians to reduce sodium intake, lose weight or make other lifestyle modifications as recommended in the guideline. ${ }^{19}$ These behaviors are likely to contribute to better control of hypertension. In a clinical setting, outpatient visit frequency may also depend on factors such as patient characteristics, understanding of hypertension, socioeconomic factors and accessibility to the clinics. It may be useful for future hypertension control to pay attention to outpatient visit frequency in light of patients' backgrounds. Hypertensive people could also be educated on the importance of appropriate outpatient visits through health intervention by insurance societies, community health centers and mass media, as well as physicians. ${ }^{28-30}$

We suspected that BP may be strongly associated with treatment during the years before 2013; therefore, we compared the effect of outpatient visits during the last 4 years of the trial (2009-2012) with that of the first 5 years (2004-2008). In fact, outpatient visits during the last 4 years (2009-2012) were more effective for hypertension control than those during the first 5 years (2004-2008). In addition, the frequency of visits during the first 5 years was also associated with BP control in 2013, even though hypertension-related factors may have changed over the years. This suggests the importance of earlier and constant outpatient visits shortly after the diagnosis of hypertension. In the present study, 36\% of the participants classified as Q4 during the first 5 years were reclassified as Q1-Q3 during the last 4 years. If the decrease in visit frequency influenced hypertension control, physicians could recommend continuing visits on an individual basis.

In the CIRCS study, the community-based intensive hypertension detection and control program, which included referral of high-risk individuals to local clinics for antihypertensive medication, was found to be both effective and cost-efficient, with a low incidence of stroke 13 years after the beginning of program. This was despite the cost of hypertension treatment and that of public health services being higher than in a control community earlier in the study. ${ }^{31}$ In another epidemiological study, Nakamura et al. ${ }^{15}$ reported that untreated grade 3 hypertension increased the risk for long-term hospitalization and incurred extremely high medical expenditure as a result of hospitalization during the year after baseline. Outpatient visit frequency is therefore important from an economic perspective.

The present study had several limitations. First, the participants were limited to the employees of a retail company. Other populations, such as community residents, should also be investigated to rule out a 'healthy worker effect' on the results. ${ }^{32}$ Second, though it is desirable to consider home $\mathrm{BP},{ }^{33,34}$ ambulatory $\mathrm{BP}^{35}$ and visit-to-visit $\mathrm{BP}$ variability to thoroughly evaluate hypertension, ${ }^{36,37}$ our study design did not allow us to consider these factors. We evaluated hypertension based on BP measurements made during health checkups in 
winter. The proportion of controlled hypertension might have been underestimated because only a single measurement was made ${ }^{38}$ and BP levels tend to be relatively higher in winter. ${ }^{9}$ Third, the analysis did not include information about the purpose of outpatient visits, BP measurement at each outpatient visit, prescriptions for antihypertensive agents and recommendations regarding lifestyle modifications.

Despite these limitations, this is the first study to show an inverse association between outpatient visit frequency and uncontrolled hypertension in a Japanese worksite population. Outpatient visit frequency was found to be a potentially important determinant of hypertension control. Greater attention and more future investigations would help in the goal of achieving better control of BP.

\section{CONFLICT OF INTEREST}

Two of the authors (AS and YK) are salaried employees of the retail company whose employees are insured by the Employees' Health Insurance Society; they provided the data for the present study. The other authors declare no conflict of interest.

\section{ACKNOWLEDGEMENTS}

This study was supported by a Grant-in-Aid for Scientific Research (C) Grant Number 25460799 from the Ministry of Education, Culture, Sports, Science and Technology of Japan.

1 Lewington S, Clarke R, Qizilbash N, Peto R, Collins R, Prospective Studies Collaboration. Age-specific relevance of usual blood pressure to vascular mortality: a meta-analysis of individual data for one million adults in 61 prospective studies. Lancet 2002; 360: 1903-1913.

2 Murakami Y, Hozawa A, Okamura T, Ueshima H, Evidence for Cardiovascular Prevention From Observational Cohorts in Japan Research Group (EPOCH-JAPAN). Relation of blood pressure and all-cause mortality in 180,000 Japanese participants: pooled analysis of 13 cohort studies. Hypertension 2008; 51: 1483-1491.

3 Turin TC, Murakami Y, Miura K, Rumana N, Kita Y, Hayakawa T, Okamura T, Okayama A, Ueshima H, NIPPON DATA80/90 Research Group. Hypertension and life expectancy among Japanese: NIPPON DATA80. Hypertens Res 2012; 35: 954-958.

4 Staessen JA, Wang JG, Thijs L. Cardiovascular prevention and blood pressure reduction: a quantitative overview updated until 1 March 2003. J Hypertens 2003; 21: 1055-1076.

5 Turnbull F, Neal B, Algert C, Chalmers J, Woodward M, MacMahon S, Blood Pressure Lowering Treatment Trialists' Collaboration. Effects of different blood-pressure-lowering regimens on major cardiovascular events: results of prospectively-designed overviews of randomised trials. Lancet 2003; 362: 1527-1535.

6 Law MR, Morris JK, Wald NJ. Use of blood pressure lowering drugs in the prevention of cardiovascular disease: meta-analysis of 147 randomised trials in the context of expectations from prospective epidemiological studies. BMJ 2009; 338: b1665.

7 Pereira M, Lunet N, Azevedo A, Barros H. Differences in prevalence, awareness, treatment and control of hypertension between developing and developed countries. J Hypertens 2009; 27: 963-975.

8 Miura K, Nagai M, Ohkubo T. Epidemiology of hypertension in Japan: where are we now? Circ J 2013; 77: 2226-2231.

9 Mori H, Ukai H, Yamamoto H, Saitou S, Hirao K, Yamauchi M, Umemura S. Current status of antihypertensive prescription and associated blood pressure control in Japan. Hypertens Res 2006; 29: 143-151.

10 Ohkubo T, Obara T, Funahashi J, Kikuya M, Asayama K, Metoki H, Oikawa T, Takahashi H, Hashimoto J, Totsune K, Imai Y, J-HOME Study Group. Control of blood pressure as measured at home and office, and comparison with physicians' assessment of control among treated hypertensive patients in Japan: First Report of the Japan Home versus Office Blood Pressure Measurement Evaluation (J-HOME) study. Hypertens Res 2004; 27: 755-763.

11 Obara T, Ohkubo T, Ishikura K, Shibamiya T, Ikeda U, Metoki H, Kikuya M, Mano N, Kuriyama S, Imai Y, J-HOME study group, J-HOME-Morning study group. Change of the management of treated hypertensive patients with or without diabetes in Japan. Clin Exp Hypertens 2013; 35: 79-86.

12 Nakamura K, Okamura T, Kanda H, Hayakawa T, Kadowaki T, Okayama A, Ueshima H, Health Promotion Research Committee of the Siga National Insurance Organizations. Impact of hypertension on medical economics: a 10-year follow-up study of national health insurance in Shiga, Japan. Hypertens Res 2005; 28: 859-864.

13 Sairenchi T, Irie F, Izumi Y, Muto T. Age-stratified analysis of the impact of hypertension on National Health Insurance Medical Expenditures in Ibaraki, Japan. J Epidemiol 2010; 20: 192-196.

14 Ohmori-Matsuda K, Kuriyama S, Hozawa A, Nakaya N, Shimazu T, Tsuji I. The joint impact of cardiovascular risk factors upon medical costs. Prev Med 2007; 44: 349-355.

15 Nakamura K, Miura K, Nakagawa H, Okamura T, Okuda N, Nishimura K, Yasumura S, Sakata K, Hidaka H, Okayama A. Treated and untreated hypertension, hospitalization, and medical expenditure: an epidemiological study in 314622 beneficiaries of the medical insurance system in Japan. J Hypertens 2013; 31: 1032-1042.

16 Ministry of Internal Affairs and Communications of Japan. Industrial Safety and Health Act (http://law.e-gov.go.jp/htmldata/S47/S47H0057.html (in Japanese)). (accessed on 20 February 2015).

17 Okamura T, Sugiyama D, Tanaka T, Dohi S. Worksite wellness for the primary and secondary prevention of cardiovascular disease in Japan: the current delivery system and future directions. Prog Cardiovasc Dis 2014; 56: 515-521.

18 Tanaka T, Okamura T, Yamagata Z, Takebayashi T, Tamura U, Kusaka Y, Urano S, Miyoshi Y, Okayama A, Ueshima H, HIPOP-OHP Research Group. Awareness and treatment of hypertension and hypercholesterolemia in Japanese workers: the High-risk and Population Strategy for Occupational Health Promotion (HIPOP-OHP) study. Hypertens Res 2007; 30: 921-928.

19 Shimamoto K, Ando K, Fujita T, Hasebe N, Higaki J, Horiuchi M, Imai Y, Imaizumi T, Ishimitsu $\mathrm{T}$, Ito $\mathrm{M}$, Ito $\mathrm{S}$, Itoh $\mathrm{H}$, Iwao $\mathrm{H}$, Kai $\mathrm{H}$, Kario $\mathrm{K}$, Kashihara $\mathrm{N}$, Kawano $\mathrm{Y}$, Kim-Mitsuyama S, Kimura G, Kohara K, Komuro I, Kumagai H, Matsuura H, Miura K, Morishita R, Naruse M, Node K, Ohya Y, Rakugi H, Saito I, Saitoh S, Shimada K, Shimosawa T, Suzuki H, Tamura K, Tanahashi N, Tsuchihashi T, Uchiyama M, Ueda S, Umemura S, Japanese Society of Hypertension Committee for Guidelines for the Management of Hypertension. The Japanese Society of Hypertension Guidelines for the Management of Hypertension (JSH 2014). Hypertens Res 2014; 37: 253-390.

20 Parchman ML, Bisonni RS, Lawler FH. Hypertension management: relationship between visit interval and control. Fam Pract Res J 1993; 13: 225-231.

21 Guthmann R, Davis N, Brown M, Elizondo J. Visit frequency and hypertension. J Clin Hypertens (Greenwich) 2005; 7: 327-332.

22 Turchin A, Goldberg SI, Shubina M, Einbinder JS, Conlin PR. Encounter frequency and blood pressure in hypertensive patients with diabetes mellitus. Hypertension 2010; 56 68-74.

23 Morrison F, Shubina M, Turchin A. Encounter frequency and serum glucose level, blood pressure, and cholesterol level control in patients with diabetes mellitus. Arch Intern Med 2011; 171: 1542-1550.

24 Ikegami N, Yoo BK, Hashimoto H, Matsumoto M, Ogata H, Babazono A, Watanabe R, Shibuya K, Yang BM, Reich MR, Kobayashi Y. Japanese universal health coverage: evolution, achievements, and challenges. Lancet 2011; 378: 1106-1115.

25 Health, Labour and Welfare Statistics Association. Trend for National Health and Hygiene, Japan 2014/2015 [in Japanese]. Health, Labour and Welfare Statistics Association: Tokyo, Japan, 2014.

26 Teramoto T, Sasaki J, Ishibashi S, Birou S, Daida H, Dohi S, Egusa G, Hiro T, Hirobe K, lida M, Kihara S, Kinoshita M, Maruyama C, Ohta T, Okamura T, Yamashita S, Yokode M, Yokote K. Comprehensive risk management for the prevention of cardiovascular disease: executive summary of the Japan Atherosclerosis Society (JAS) guidelines for the diagnosis and prevention of atherosclerotic cardiovascular diseases in Japan - 2012. J Atheroscler Thromb 2013; 20: 603-615.

27 The Japan Diabetes Society. Evidence-based Practice Guideline for the Treatment of Diabetes in Japan 2013. Nankodo: Tokyo, Japan, 2013.

28 Jaffe MG, Lee GA, Young JD, Sidney S, Go AS. Improved blood pressure control associated with a large-scale hypertension program. JAMA 2013; 310: 699-705.

29 Miyamatsu N, Okamura T, Nakayama H, Toyoda K, Suzuki K, Toyota A, Hata T, Hozawa A, Nishikawa T, Morimoto A, Ogita M, Morino A, Yamaguchi T. Public awareness of early symptoms of stroke and information sources about stroke among the general Japanese population: the Acquisition of Stroke Knowledge Study. Cerebrovasc Dis 2013; 35: 241-249.

30 Morimoto A, Miyamatsu N, Okamura T, Nakayama H, Toyoda K, Suzuki K, Toyota K, Hata T, Yamaguchi T. Effect of intensive and moderate public education on knowledge of early stroke symptoms among Japanese population: the Acquisition of Stroke Knowledge Study. Stroke 2013; 44: 2829-2834.

31 Yamagishi K, Sato S, Kitamura A, Kiyama M, Okada T, Tanigawa T, Ohira T, Imano H, Kondo M, Okubo I, Ishikawa Y, Shimamoto $\mathrm{T}$, Iso H, CIRCS Investigators. Cost-effectiveness and budget impact analyses of a long-term hypertension detection and control program for stroke prevention. Hypertens Res 2013; 30: 1874-1879.

$32 \mathrm{Li} \mathrm{CY}$, Sung FC. A review of the healthy worker effect in occupational epidemiology. Occup Med (Lond) 1999; 49: 225-229.

33 Sakuma M, Imai Y, Nagai K, Watanabe N, Sakuma H, Minami N, Satoh H, Abe K. Reproducibility of home blood pressure measurements over a 1-year period. Am J Hypertens 1997: 10: 798-803.

34 Asayama K, Ohkubo T, Metoki H, Obara T, Inoue R, Kikuya M, Thijs L, Staessen JA, Imai $Y$, Hypertension Objective Treatment Based on Measurement by Electrical devices of Blood Pressure (HOMED BP). Cardiovascular outcomes in the first trial of antihypertensive therapy guided by self-measured home blood pressure. Hypertens Res 2012; 35: 1102-1110.

35 Pickering TG, Shimbo D, Haas D. Ambulatory blood-pressure monitoring. N Engl J Med 2006; 354: 2368-2374.

36 Faramawi MF, Delongchamp R, Said Q, Jadhav S, Abouelenien S. Metabolic syndrome is associated with visit-to-visit systolic blood pressure variability in the US adults. Hypertens Res 2014; 37: 875-879.

37 Nakano C, Morimoto S, Nakahigashi M, Kusabe M, Ueda H, Someya K, Ichihara A, Iwasaka T, Shiojima I. The relationships between visit-to-visit blood pressure variability and renal and endothelial function in chronic kidney disease. Hypertens Res 2015; 38 193-198.

38 MacMahon S, Peto R, Cutler J, Collins R, Sorlie P, Neaton J, Abbott R, Godwin J, Dyer A, Stamler J. Blood pressure stroke and coronary heart disease. Part 1, Prolonged differences in blood pressure: prospective observational studies corrected for the regression dilution bias. Lancet 1990; 335: 765-774. 